\title{
Lusioersily
}

\section{Change of Structural Behaviors of Organo-Silane Exposed Graphene Nanoflakes}

Pao, CW., Ray, SC., Tsai, HM., Chen, HC., Lin, IN., Pong, WF., Chiou, JW., Tsai, MH., Shang, NG.,

Papakonstantinou, P., \& GuO, GH. (2010). Change of Structural Behaviors of Organo-Silane Exposed Graphene Nanoflakes. Journal Of Physical Chemistry C, 114, 8161-8166. https://doi.org/10.1021/jp9121563

Link to publication record in Ulster University Research Portal

\section{Published in:}

Journal Of Physical Chemistry C

Publication Status:

Published (in print/issue): 01/04/2010

DOI:

10.1021/jp9121563

\section{Document Version}

Publisher's PDF, also known as Version of record

\section{General rights}

Copyright for the publications made accessible via Ulster University's Research Portal is retained by the author(s) and / or other copyright owners and it is a condition of accessing these publications that users recognise and abide by the legal requirements associated with these rights.

\section{Take down policy}

The Research Portal is Ulster University's institutional repository that provides access to Ulster's research outputs. Every effort has been made to ensure that content in the Research Portal does not infringe any person's rights, or applicable UK laws. If you discover content in the Research Portal that you believe breaches copyright or violates any law, please contact pure-support@ulster.ac.uk. 


\title{
Change of Structural Behaviors of Organo-Silane Exposed Graphene Nanoflakes
}

\author{
C. W. Pao,${ }^{\dagger}$ S. C. Ray, ${ }^{*}{ }^{\dagger},{ }^{\dagger}$ H. M. Tsai,${ }^{\dagger}$ Y. S. Chen, ${ }^{\dagger}$ H.-C. Chen, ${ }^{\dagger}$ I.-N. Lin, ${ }^{\dagger}$ W. F. Pong, ${ }^{*}, \dagger$ \\ J. W. Chiou, ${ }^{\S}$ M.-H. Tsai, ${ }^{\|}$N. G. Shang, ${ }^{\perp}$ P. Papakonstantinou, ${ }^{\perp}$ and J.-H. Guo ${ }^{\#}$ \\ Department of Physics, Tamkang University, Tamsui 251, Taiwan, School of Physics, University of the \\ Witwatersrand, Private Bag 3, WITS 2050, Johannesburg, South Africa, Department of Applied Physics, \\ National University of Kaohsiung, Kaohsiung 811, Taiwan, Department of Physics, National Sun Yat-Sen \\ University, Kaohsiung 804, Taiwan, Nanotechnology and Integrated Bio-Engineering Centre, University of \\ Ulster, Shore Road, Newtownabbey, BT37 OQB, United Kingdom, and Advanced Light Source, Lawrence \\ Berkeley National Laboratory, Berkeley, California 94720
}

Received: December 24, 2009; Revised Manuscript Received: March 30, 2010

\begin{abstract}
The electronic structures of graphene nanoflakes (GNFs) exposed to an organo-silane precursor [tetramethylsilane, TMS, $\mathrm{Si}\left(\mathrm{CH}_{3}\right)_{4}$ ] were studied using electron field emission (EFE), Raman spectroscopy, X-ray absorption near-edge structure (XANES), X-ray photoelectron spectroscopy (XPS), X-ray emission spectroscopy (XES), and first-principles calculation. The results of XANES, XPS, and Raman spectroscopy indicate that the silyl radical strong covalent bonds were formed in GNFs, which induced local structural relaxations and enhanced $\mathrm{sp}^{3}$ hybridization. Comparison of calculated electronic structure, XANES, and XES spectra of Sitreated GNFs suggests that the $\mathrm{Si}$ atom substitutes one 3-fold coordinated $\mathrm{C}$ atom in a given graphene layer and relaxes outward to form $\mathrm{sp}^{3}$ bonding with another $\mathrm{C}$ atom in the adjacent graphene layer. The EFE measurements show an increase in the turn-on electric field with the increase of the Si content, which suggests an enhancement of the nonmetallic $\mathrm{sp}^{3}$ bonding.
\end{abstract}

\section{Introduction}

Graphene nanoflakes (GNFs) are quasi-two-dimensional networks of vertically aligned graphene sheets ${ }^{1}$ and have morphologies similar to those of nanocarbon materials, such as carbon nanoflakes ${ }^{2,3}$ and nanosheets. ${ }^{4}$ The thicknesses of GNFs range from 1 to $2 \mathrm{~nm}$ (several graphene layers) at the edges to $20 \mathrm{~nm}$ at the base. The sharp edges of GNFs are responsible for low-threshold field emission characteristics, making them useful as backlights in liquid crystal displays ${ }^{5}$ and in highbrightness lamps in the form of wires coated with nickel. ${ }^{6}$ With a combination of edge plane reactivity, special nestlike morphology, and large surface area, GNFs present a distinct opportunity for the creation of a new class of electrodes with a wide range of applications in the electroanalytical, biosensing, and energy storage/conversion sectors ${ }^{7}$ similar to those of carbon nanotubes (CNTs). Despite these potential applications, GNFs, like graphite, are soft and hence mechanically unstable. Silicon is a highly reactive center that creates opportunities for silylated GNFs (GNFs:Si) to be used as novel nanodevices, such as chemical sensors and toxic gas scrubbers. ${ }^{8}$ On the basis of theoretical studies, substitutionally doped $\mathrm{Si}$ atoms in CNTs were found to relax outward and form $\mathrm{sp}^{3}$ bonding. ${ }^{9,10}$ The formation of $\mathrm{sp}^{3}$ bonding in GNFs:Si can be expected to enhance their mechanical stability. Si atoms chemisorbed on the surfaces of GNFs or CNTs provide dangling bonds, which may mediate the formation of other types of materials. On the basis of firstprinciples calculations, Song et al. found that Si dopants in CNTs

* To whom correspondence should be addressed. E-mail: raysekhar@ rediffmail.com (S.C.R.), wfpong@mail.tku.edu.tw (W.F.P.).

$\dagger$ Tamkang University.

University of the Witwatersrand.

$\S$ National University of Kaohsiung.

"National Sun Yat-Sen University.

${ }^{\perp}$ University of Ulster.

\# Lawrence Berkeley National Laboratory. behaved as a binding center for preferential attachment of various atoms or molecules for further graphene functionalization. ${ }^{11} \mathrm{Si}$ is preferable to other group IV elements because their much larger sizes can distort greatly the host network. To understand the atomic arrangement of $\mathrm{Si}$ dopants and the formation of an $\mathrm{sp}^{3}$-bonding arrangement in graphene networks, a combination of Raman spectroscopy, X-ray absorption nearedge structure (XANES), X-ray photoelectron spectroscopy (XPS), and X-ray emission spectroscopy (XES) measurements and first-principles calculation have been performed. The present experimental measurements and theoretical calculation show that Si treating indeed enhances the $\mathrm{sp}^{3}$-bonding arrangement through the formation of a local tetrahedral atomic arrangement.

\section{Experimental Details}

The C K-edge XANES and core-level XPS spectra were measured using the high-energy spherical grating monochromator and low-energy spherical grating monochromator beamlines, respectively, with an electron energy of $1.5 \mathrm{GeV}$ and a maximum stored current of $200 \mathrm{~mA}$ at the National Synchrotron Radiation Research Center, Hsinchu, Taiwan. The XANES spectra were obtained in the sample drain current mode, whereas the core-level XPS spectra were obtained at an excitation energy of $374.2 \mathrm{eV}$, with a typical resolution of $0.1 \mathrm{eV}$ at a base pressure of $\sim 5 \times 10^{-10}$ Torr. The $\mathrm{C} \mathrm{K \alpha}$ XES measurements were made at beamline-7.0.1 at the Advanced Light Source, Lawrence Berkeley National Laboratory. The resolution of the XES measurement was set to $\sim 0.35 \mathrm{eV}$. C K-edge XANES spectra are normalized using incident beam intensity $I_{\mathrm{o}}$ following pre-edge background subtraction and by keeping the area under the spectra in the energy range fixed between 315 and $330 \mathrm{eV}$ (not shown in the figure). The electron field emission (EFE) measurements for pure GNFs and GNFs:Si were performed using a Keithley power supply. Raman spectroscopy was 


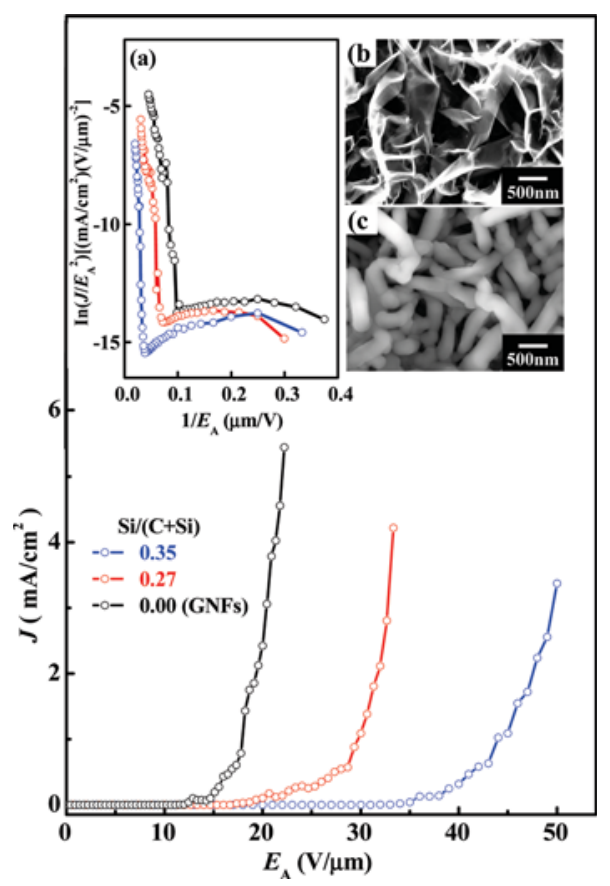

Figure 1. Electron emission current density $(J)$ as a function of applied electrical field $\left(E_{\mathrm{A}}\right)$ of GNFs and GNF:Si at various $\mathrm{Si}$ atom \%. The inset (a) shows electron field emission $\mathrm{F}-\mathrm{N}$ plots: $\left(1 / E_{\mathrm{A}}\right)$ vs $\left(J / E_{\mathrm{A}}{ }^{2}\right)$ of GNFs and GNFs:Si at various Si atom \%. Insets (b) and (c) show SEM images of GNFs and GNFs: $\mathrm{Si}$ with $\mathrm{Si} / \mathrm{Si}+\mathrm{C}=0.35$, respectively.

performed with an excitation energy of $514 \mathrm{~nm}(2.41 \mathrm{eV})$ and a spectral resolution better than $2 \mathrm{~cm}^{-1}$. GNFs with a thickness of $\sim 1250 \mathrm{~nm}$ were synthesized directly on the $\mathrm{Si}$ substrate without catalyst by the microwave-plasma enhanced chemical vapor deposition (CVD) method and were subsequently subjected to RF-plasma glow discharge with various Ar-totetramethylsilane [TMS, $\left.\mathrm{Si}\left(\mathrm{CH}_{3}\right)_{4}\right]$ ratios at room temperature for $5 \mathrm{~min}^{7}$

\section{Results and Discussion}

Figure 1 plots the EFE current density $(J)$ as a function of the applied electric field $\left(E_{\mathrm{A}}\right)$. This figure shows the existence of a threshold electric field, at which $J$ increases from zero significantly. This threshold electric field, in general, increases with the increase of the $\mathrm{Si}$ content, which suggests that $\mathrm{Si}$ treatment increases the activation energy. For a given GNFs:Si sample, $J$ increases roughly exponentially beyond the threshold field. To further demonstrate the existence of a threshold electric field or turn-on electric field ( $\left.E_{\mathrm{TOE}}\right)$, Fowler-Nordheim $(\mathrm{F}-\mathrm{N})$ plots are shown in inset (a) of Figure 1. $E_{\mathrm{TOE}}$ were obtained with linear curve fitting in the high electric field region and were found to increase from $9.8 \mathrm{~V} / \mu \mathrm{m}$ for pure GNFs to 26.3 V/ $\mu \mathrm{m}$ for GNFs:Si with the highest $\mathrm{Si}$ content $(\mathrm{Si} /(\mathrm{Si}+\mathrm{C})=$ 0.35 ) by the formation of more $\mathrm{sp}^{3}$-hybridized bonds in GNFs: Si because $\mathrm{Si}$ prefers $\mathrm{sp}^{3}$ than $\mathrm{sp}^{2}$ bonding, unlike $\mathrm{C}$ (which prefers $\mathrm{sp}^{2}$ bonding). Insets (b) and (c) of Figure 1 present scanning electron microscopic (SEM) images of pure GNFs and GNFs:Si with the highest $\mathrm{Si}$ content $(\mathrm{Si} / \mathrm{Si}+\mathrm{C}=0.35)$, respectively. Pure GNFs shown in inset (b) contain petal-like graphitic nanoflakes with very sharp edges, which are randomly interlaced to form a nestlike porous structure with a large surface area, ${ }^{7}$ whereas inset (c) shows round nanoflakes in the form of rod- or tubelike GNFs:Si. The strong preference for $\mathrm{sp}^{3}$ bonding was argued to be related to an outward relaxation of the $\mathrm{Si}$ atom. ${ }^{9,10}$ Therefore, reduction of the EFE current in GNFs:Si is

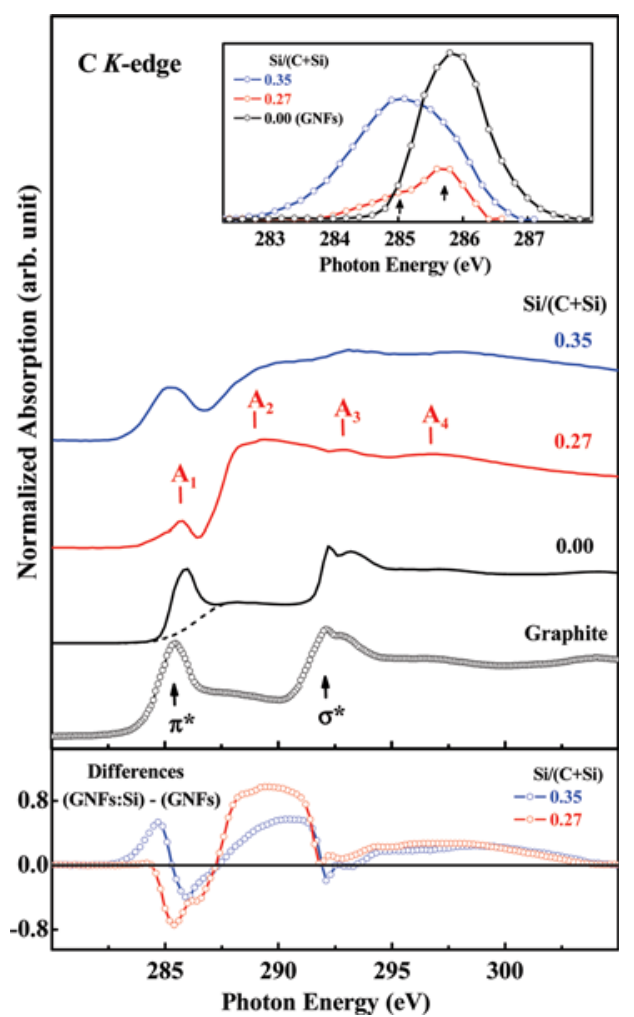

Figure 2. Normalized C K-edge XANES spectra of GNFs, Si-treated GNFs at various $\mathrm{Si}$ atom \%, and reference graphite. The upper inset presents the magnified $\pi^{*}$ region of the C K-edge XANES spectra of GNFs following subtraction by a best-fitted Gaussian line (dashed line). The bottom panel shows difference XANES spectra of Si-treated GNFs and GNFs.

due to the decrease of the number of $\mathrm{sp}^{2}$ hybridized bonds in the network of GNFs:Si because $\mathrm{sp}^{2}$-hybridized bonds in GNFs are responsible for the metallic EFE current. ${ }^{12}$

Figure 2 displays the C K-edge XANES spectra of GNFs, GNFs:Si, and reference graphite. These spectra reflect transition from the $\mathrm{C} 1 \mathrm{~s}$ core state to the $\mathrm{p}$-like final states above the Fermi level $\left(E_{\mathrm{f}}\right)$. The $\pi^{*}$ features associated with $\mathrm{sp}^{2}$-bonding configurations were observed at $\sim 285.5$ and $285.8 \mathrm{eV}$ in the XANES spectra of graphite and GNFs (marked as $\mathrm{A}_{1}$ ), respectively, ${ }^{13,14}$ whereas the $\sigma^{*}$ features were observed at $\sim 293$ $\mathrm{eV}$. The upper inset of Figure 2 shows that GNFs:Si exhibit double structural features (indicated by two vertical arrows) centered at $\sim 285.0$ and $285.7 \mathrm{eV}$ in the $\pi^{*}$ region, whose intensities increase gradually with the $\mathrm{Si}$ content. The center of the $\pi^{*}$ feature shifts from $\sim 285.7 \mathrm{eV}$ for pure GNFs to the lower energy of $\sim 285.0 \mathrm{eV}$ for GNFs:Si with the highest ( $\mathrm{Si}$ / $\mathrm{Si}+\mathrm{C}$ ) ratio $=0.35$. A similar downward energy shift of $\sim 0.7$ $\mathrm{eV}$ has been observed in Si-treated diamond-like carbon films. ${ }^{14}$ The double features in the $\pi^{*}$ region show the possibility of the formation of two kinds of bonds, namely, $\mathrm{Si}-\mathrm{C}(\mathrm{:H}) / \mathrm{Si}-\mathrm{C}($ : $\mathrm{O})$ and $\mathrm{sp}^{2} \mathrm{C}-\mathrm{C}$ bonds, which were also observed by Terekhov et al. in a XANES study of $a-\mathrm{Si}_{0.4} \mathrm{C}_{0.6}: \mathrm{H}$ composite materials. ${ }^{15}$ The general line shape of the $\mathrm{C}$ K-edge XANES spectrum of $\mathrm{SiC} / \beta-\mathrm{SiC}^{15,16}$ is clearly different from that of GNFs:Si, which indicates the absence of SiC segregations in GNFs:Si. However, the intensity of the $\pi^{*}$ feature decreases significantly for $(\mathrm{Si} / \mathrm{Si}$ $+\mathrm{C})$ ratio $=0.27$ and the $\pi^{*}$ feature becomes wider for ( $\mathrm{Si} / \mathrm{Si}$ $+\mathrm{C}$ ) ratio $=0.35$, as clearly seen in Figure 2 (upper inset). Similarly broadened double peaks were also observed in CVD diamond (see ref 15, Figure 6). On comparison with the spectral features of the CVD diamond, ${ }^{15}$ GNFs:Si can be concluded to contain an increased number of $\mathrm{sp}^{3}$-hybridized atoms with 
diamond-like character, consistent with C 1s core-level XPS results to be discussed later. However, in the spectra of GNFs: $\mathrm{Si}$, the $\sigma^{*}$ feature essentially disappears and is replaced by a very broad feature with the leading edge located around 288 $\mathrm{eV}$ for $\mathrm{Si} /(\mathrm{Si}+\mathrm{C})=0.27 \mathrm{GNFs}: \mathrm{Si}$. As for $\mathrm{Si} /(\mathrm{Si}+\mathrm{C})=0.35$ GNFs:Si, this leading edge is further broadened. Beyond the $\sim 288 \mathrm{eV}$ leading edge, which is marked as $\mathrm{A}_{2}$ in the spectrum of $\mathrm{Si} /(\mathrm{Si}+\mathrm{C})=0.27 \mathrm{GNFs}: \mathrm{Si}$, there are two extremely shallow and broad features marked as $\mathrm{A}_{3}$ and $\mathrm{A}_{4}$. Note that Pacilié et al. recently observed a feature located at $\sim 288 \mathrm{eV}$ for single and few-layer graphenes and attributed it to the interlayer state. ${ }^{17}$ Because this $\sim 288 \mathrm{eV}$ feature is absent in the spectra of graphite and pure GNFs, it could be due to a shortened or elongated interlayer spacing ${ }^{18}$ and has a different origin from feature $\mathrm{A}_{2}$ observed for GNFs:Si. The XPS compositional analysis also indicates the presence of the oxygen content, which increases from 2 atom \% in pure GNFs to 24-38 atom \% in GNFs:Si. Thus, there is a possibility of the formation of the $\mathrm{Si}-\mathrm{C}(: \mathrm{O})$ bonding in addition to the $\mathrm{Si}-\mathrm{C}(: \mathrm{H})$ bonding.

The spectral difference between pure GNFs and GNFs:Si shown in the lower inset of Figure 2 reveals the influence of $\mathrm{Si}-\mathrm{C}(: \mathrm{H})$ and/or $\mathrm{Si}-\mathrm{C}(: \mathrm{O})$ bonding on the electronic structure of GNFs. In the $\pi^{*}$ region presented in the lower inset, the difference spectra are negative between about 284 and $287 \mathrm{eV}$ for $\mathrm{Si} /(\mathrm{Si}+\mathrm{C})$ ratio $=0.27$, while in this region, the difference spectrum rises up and then decreases to become negative for $\mathrm{Si} /(\mathrm{Si}+\mathrm{C})$ ratio $=0.35$. On the other hand, in the region between 287 and $292 \mathrm{eV}$, all difference spectra are positive for the two $\mathrm{Si} /(\mathrm{Si}+\mathrm{C})$ ratios and the intensity decreases with the increase of the $\mathrm{Si} /(\mathrm{Si}+\mathrm{C})$ ratio. This trend seems opposite to what one would expect because one would expect that the deviation of these features from those of pure GNFs should increase with the Si content. However, one may interpret the effect of $\mathrm{Si}$ dopants by the shift of some states from the 284-287 eV regions to the higher-energy 287-292 eV region. If one subtracts the area under the positive difference intensities, which is a measure of the number of states, by that of the negative difference intensities, the two GNFs:Si samples have net gains of states in the $284-292 \mathrm{eV}$ regions. The positive broad features in the 292-305 eV range are similar for GNFs: $\mathrm{Si}$ considered in this study. Overall, Si treating enhances the number of near-edge unoccupied $\mathrm{C} 2 \mathrm{p}$ derived states.

Figure 3a shows that Raman spectra of pure GNFs and GNFs: $\mathrm{Si}$ are similar and both have a D band, which is a characteristic of either a disordered cluster or a small crystallite, and a $\mathrm{G}$ band, which can be attributed to the stretching vibration mode $\mathrm{E}_{2 \mathrm{~g}}$ of single-crystal graphite. ${ }^{19}$ In addition, a $\mathrm{D}^{\prime}$ band, which was attributed to symmetry breaking in the microscopic $\mathrm{sp}^{2}$ crystallite, appears as a shoulder of the $\mathrm{G}$ band. ${ }^{20}$ It suggests that there is no significant SiC segregations or clusters in GNFs:Si. $D$ and $G$ bands are shifted from $1337 \mathrm{~cm}^{-1}$ (GNFs) to 1331 $\mathrm{cm}^{-1}$ (GNFs:Si) and 1585 to $1580 \mathrm{~cm}^{-1}$, respectively. Broadening of these bands indicates the formation of a larger number of $\mathrm{sp}^{3}$-hybridized atoms in GNFs:Si. Bands in the Raman spectra correspond to vibration frequencies, which are proportional to the square root of the force constant of the stretching or bending vibration. The $\mathrm{D}, \mathrm{G}$, and $\mathrm{D}^{\prime}$ bands with frequencies on the order of a thousand $\mathrm{cm}^{-1}$ are due to stretching vibrations of the $\mathrm{C}-\mathrm{C}$ bond in different normal modes. The force constant is proportional to the curvature or second derivative of the stretching potential well and is usually larger for a deeper potential well or a greater bond strength. Thus, the slight decrease of the frequencies of $\mathrm{D}$ and $\mathrm{G}$ bands of $\mathrm{Si}$-doped graphene flakes reflects a slight weakening of the $\mathrm{C}-\mathrm{C}$ bond caused by bonding (a)

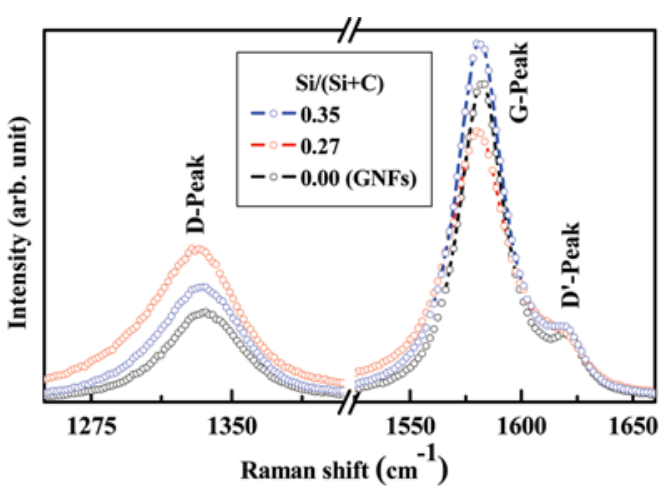

(b)

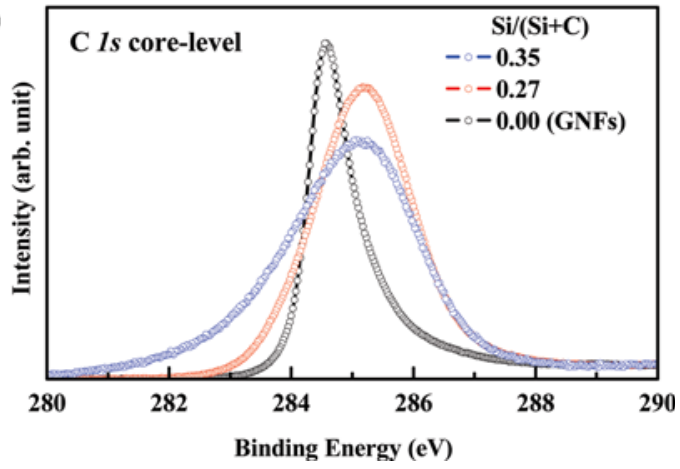

Figure 3. (a) Raman spectra of GNFs and Si-treated GNFs and (b) C 1s core-level XPS spectra of GNFs and Si-treated GNFs.

with the Si atom. This observation is an indication of Si-dopantinduced partial conversion of the $\mathrm{sp}^{2}$ bonding, which is a mixture of single and double $\mathrm{C}-\mathrm{C}$ bonds, to the $\mathrm{sp}^{3}$ bonding with only single $\mathrm{C}-\mathrm{C}$ bonds. Figure $3 \mathrm{~b}$ shows $\mathrm{C} 1 \mathrm{~s}$ core-level XPS spectra. The peak observed at $\sim 284.4 \mathrm{eV}$ for GNFs can be assigned as the $\mathrm{C}-\mathrm{C}$ bond, which is shifted to the higher energy at $\sim 285.2 \mathrm{eV}$ and became broader for GNFs:Si. Pedio et al. ${ }^{21}$ studied the dependence of the formation of $\mathrm{C}_{60}$ on silicon surfaces on the annealing temperature using C 1s XPS measurements and found that the $\mathrm{C}-\mathrm{C}$ bond and $\mathrm{C}-\mathrm{Si}$ bond features are located at 284.2 and $282.6 \mathrm{eV}$, respectively. In the present case, the spectral features are different and do not have the signature of $\mathrm{SiC}$ segregations in $\mathrm{C}$ 1s core-level XPS spectra, which further confirms enhanced $\mathrm{sp}^{3}$ bonding (i.e., diamond and/ or diamond-like carbon) rather than $\mathrm{sp}^{2}$ bonding (like graphite and/or CNTs) and/or $\mathrm{C}-\mathrm{Si}$ bonding. This result is consistent with those obtained from Raman and XANES measurements. The enhancement of $\mathrm{sp}^{3}$ bonding and reduction of $\mathrm{sp}^{2}$ bonding improves the reaction activity of GNFs:Si because surface atoms with $\mathrm{sp}^{3}$ bonding have dangling bonds.

Figure 4 presents C K $\alpha$ XES spectra of GNFs, GNFs:Si, and reference graphite obtained at an excited energy $\left(E_{\mathrm{ex}}\right)$ of 320 $\mathrm{eV}$. The left upper part of the inset in Figure 4 presents the experimental geometry used in this work, in which $E$ is the polarization of the incoming photons and $\theta$ is the incidence angle. The spectral features of graphite shown in Figure 4 are consistent with reports available in the literature. ${ }^{22,23}$ The shape of the $\mathrm{C} \mathrm{K} \alpha$ XES spectrum of graphite was found to depend strongly on both incident angle and excitation energy due to the different intensity distribution between $\pi$ and $\sigma$ components of the emission. ${ }^{22}$ For XES measurement, the two features near the $277-284$ and $270-277 \mathrm{eV}$ regions of graphite are known to correspond to occupied $\pi$ and $\sigma$ bands, which are derived from the transition from $2 \mathrm{p}_{\pi}$ and $2 \mathrm{p}_{\sigma}$ states oriented parallel and perpendicular to the graphite plane, respectively. The spectra of pure GNFs and GNFs:Si can be divided into $\mathrm{p}_{\sigma}$ and $\mathrm{p}_{\pi}$ bands 


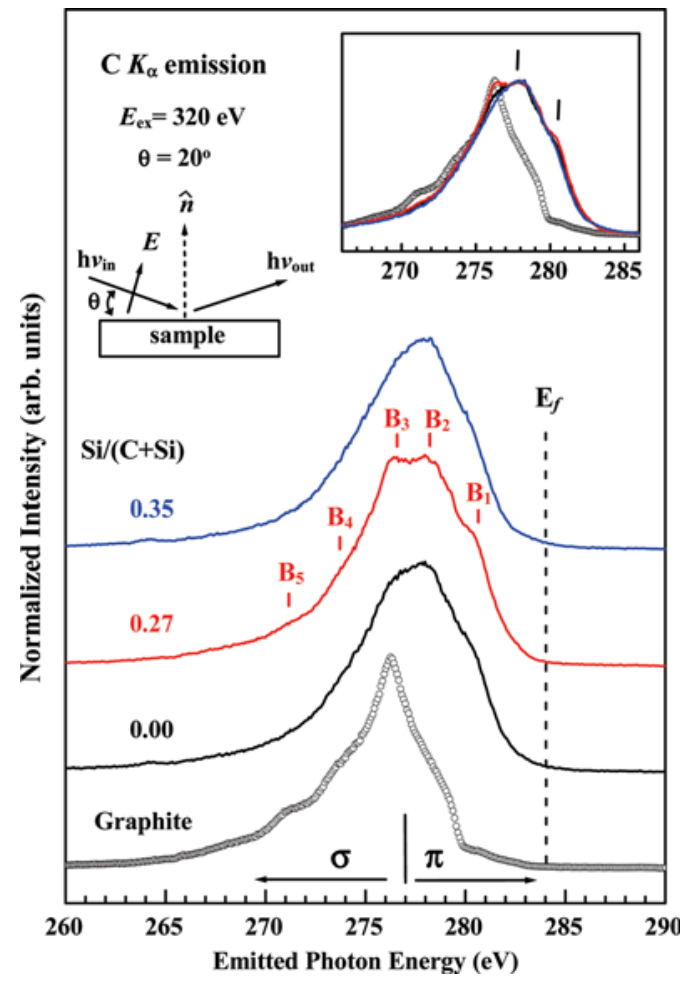

Figure 4. $\mathrm{C} \mathrm{K} \alpha$ XES spectra of GNFs, Si-treated GNFs, and reference graphite obtained at an excited energy of $320 \mathrm{eV}$. The left upper part of the inset presents the experimental geometry, in which $E$ is the polarization of the incoming photons, $\theta\left(=20^{\circ}\right)$ is the incidence angle, and the Fermi level is indicated as $E_{\mathrm{f}}$. The right upper inset highlights the $\pi$ and $\sigma$ regions of $\mathrm{C} \mathrm{K \alpha}$ XES spectra.

centered at $\sim 278$ and $281 \mathrm{eV}$, respectively, as indicated by the two solid bars in the right upper inset (Figure 4). These two bands shift toward $E_{\mathrm{f}}$, and the overall intensities are greatly enhanced relative to those of graphite, as shown in the right upper inset. Though the details of the $\mathrm{p}_{\sigma}$ band are quite sensitive to the $\mathrm{Si} /(\mathrm{C}+\mathrm{Si})$ ratio, $\mathrm{Si}$ treatment does not affect significantly the overall intensity of the valence band of GNFs:Si, which implies that $\mathrm{Si}$-derived p states lie dominantly above $E_{\mathrm{f}}$; this is supported by the present calculation result. The drastic difference between the spectra of pure GNFs and grahite, both of which are composed of graphene layers, can be caused by the random nestlike porous structure of GNFs, as shown in inset (b) of Figure 1, because the shapes of $\mathrm{C} \mathrm{K} \alpha$ XES spectra depend strongly on the incident angle of photons. ${ }^{22}$

\section{Theoretical Calculation}

To elucidate the bonding arrangement of Si dopants in GNFs: $\mathrm{Si}$, first-principles calculations of the electronic structure of GNFs:Si with a 25 atom \% of $\mathrm{Si}$ have been performed. The similarity between $\mathrm{C}$ K-edge XANES spectra of pure GNFs and graphite, as shown in Figure 2, indicates that the electronic structures of GNFs:Si are bulk-like so that a bulk model is adequate for GNFs:Si. By analogy with previous theoretical studies of Si-doped nanotubes ${ }^{9-11}$ and that $\mathrm{Si}$ dopants were found to favor the formation of a 4-fold coordinated tetrahedral bonding arrangement, ${ }^{8}$ here, $\mathrm{Si}$ dopants are assumed to substitute for $\mathrm{C}$ atoms in the graphene layers not trapped between the graphene layers and that the $\mathrm{Si}$ dopant is displaced vertically from the graphene plan so that it forms a 4-fold bonding arrangement with three $\mathrm{C}$ atoms ${ }^{24}$ in one graphene layer (see Figure 5) and another $\mathrm{C}$ atom in the adjacent $\mathrm{AB}$-stacked graphene layer. It is well-known that the coupling strength

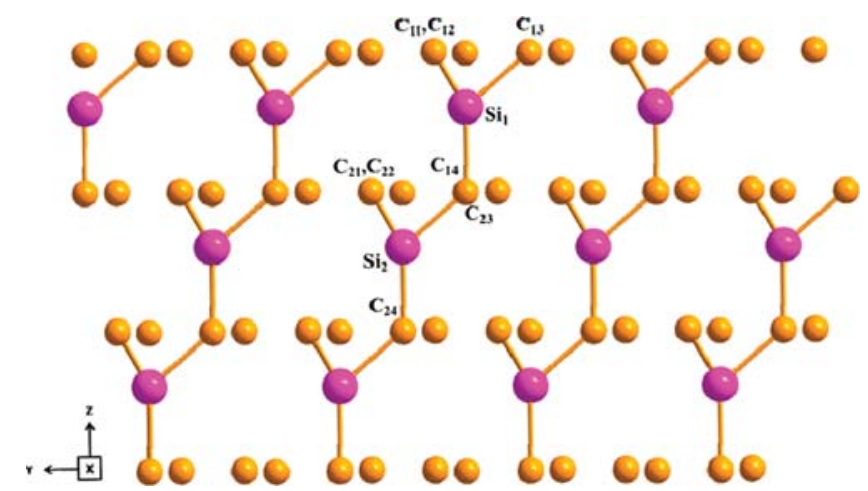

Figure 5. Side view of the structural model of Si-treated GNFs. The $z$ axis is perpendicular to the graphene plane, and the $y$ axis is in the [1010] direction. In the figure, $\mathrm{C}$ atoms $\mathrm{C}_{11}, \mathrm{C}_{12}, \mathrm{C}_{13}$, and $\mathrm{C}_{14}$ are bonded with $\mathrm{Si}$ atom $\mathrm{Si}_{1}$, while $\mathrm{C}_{21}, \mathrm{C}_{22}, \mathrm{C}_{23}$, and $\mathrm{C}_{24}$ are bonded with $\mathrm{Si}_{2}$. Note that $\mathrm{C}_{11}$ and $\mathrm{C}_{12}\left(\mathrm{C}_{21}\right.$ and $\left.\mathrm{C}_{22}\right)$ coincide in the $(y, z)$ plane and $\mathrm{C}_{14}$ and $\mathrm{C}_{23}$ refer to different $\mathrm{C}$ atoms, though they appear to be the same in the figure.

between two adjacent graphene layers is very weak and is negligible. The substitution of a carbon atom in the graphene layer by a $\mathrm{Si}$ atom, which is bonded to three $\mathrm{C}$ atoms in the given graphene layer and a $\mathrm{C}$ atom in the adjacent layer, in the structural model proposed in this study is to replace three $\mathrm{C}-\mathrm{C}$ bonds by four single $\mathrm{Si}-\mathrm{C}$ bonds. Because $\mathrm{Si}$ is not likely to form a graphite-like planar structure with a mixing of single and double bonds, it is expected that the coordination with four $\mathrm{C}$ atoms is more favorable than with three or fewer $\mathrm{C}$ atoms for the Si impurity atom. Natural bulk graphite can be composed of graphene layers with $\mathrm{AB}$ and $\mathrm{AA}$ stacking sequences with the $\mathrm{AB}$ stacking sequence the most common; that is, the $\mathrm{AB}$ stacking sequence is more favorable than the AA stacking sequence. With a 0.25 atom $\% \mathrm{Si} /(\mathrm{C}+\mathrm{Si})$ composition and a $(2 \times 2)$ unit cell, Si dopants will form a two-dimensional array of $\mathrm{Si}$ chains if an $\mathrm{ABAB}$ stacking sequence with a two-graphenelayer periodicity along the $c$ axis is chosen. To better disperse $\mathrm{Si}$ atoms in order to represent better the randomly distributed Si dopants, an $\mathrm{ABCABC}$ stacking sequence is chosen. Note that ABCABC stacking does not have any AA stacking between any two adjacent graphene layers and is structurally similar to the ABAB stacking. Note that the GNFs:Si sample with a ratio closest to this ratio is $\mathrm{Si} /(\mathrm{C}+\mathrm{Si})=0.27$. To choose a 0.27 ratio requires a very large unit cell; a ratio of 0.25 has a relatively small and manageable unit cell with $21 \mathrm{C}$ atoms and $3 \mathrm{Si}$ atoms. A total energy minimization or relaxation procedure has been used to obtain the optimized lattice constant, $a$, graphene layer spacing, and the $\mathrm{Si}-\mathrm{C}$ bond length of $2.408,3.095$, and 1.86 $\AA$, respectively. The $\mathrm{Si}-\mathrm{C}$ bond length is close to the sum of their covalent radii of $1.88 \AA .{ }^{25}$ The lattice constant is about $2 \%$ smaller than the experimental one of the bulk graphite of $2.460 \AA,{ }^{26}$ whereas the layer spacing is significantly shortened relative to the theoretical one of graphite of $3.257 \AA^{18}$ due to the formation of $\mathrm{Si}-\mathrm{C}$ tetrahedral bonding. In this proposed structural model, Si dopants are arranged in an orderly manner.

The modified pseudofunction first-principles method ${ }^{27}$ is employed to calculate the electronic structure and partial densities of states (PDOS) of the 25 atom \% GNFs:Si. The basis set contains Bloch sums of muffin-tin orbitals with spherical Hankel and Neumann tailing functions. This calculation method has been shown to work well for graphite and few-layer graphenes. ${ }^{18}$ The 12 special $k$-points of Cohen and Chadi for a hexagonal lattice ${ }^{28}$ are used to approximate the integration over the first Brillouin zone to obtain self-consistent potentials. The Monkhurst-Pack special $k$-point scheme ${ }^{29}$ with $q$ values of 9 , 

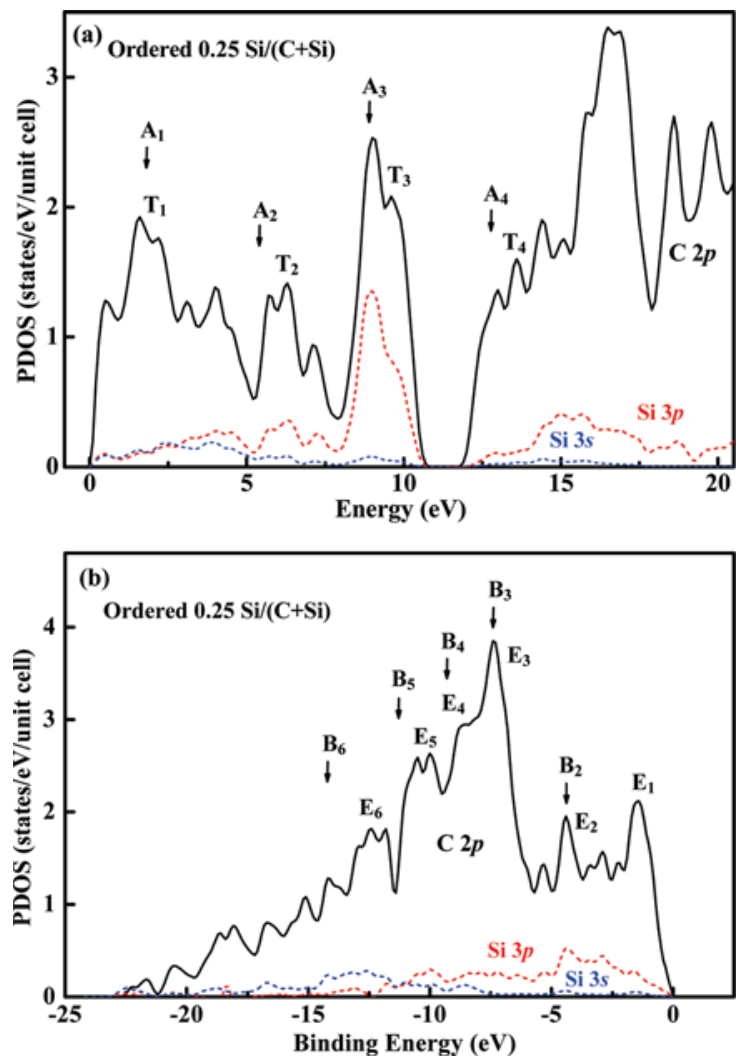

Figure 6. (a) Calculated PDOS of C $2 p$ (solid line) and Si $3 s$ and $3 p$ (dashed lines) states of the 25 atom \% Si-treated GNFs above the Fermi level. (b) Calculated PDOS of occupied C 2p and Si 3s and 3p states.

9, and 3 is used to obtain the PDOS. Figure 6a shows the PDOS of $\mathrm{C} 2 \mathrm{p}, \mathrm{Si} 3 \mathrm{~s}$, and $\mathrm{Si} 3 \mathrm{p}$ states above $E_{\mathrm{f}}$. Features are divided into $\mathrm{T}_{1}, \mathrm{~T}_{2}, \mathrm{~T}_{3}$, and $\mathrm{T}_{4}$ groups in the calculated PDOS of $\mathrm{C} 2 \mathrm{p}$ states. These four groups can be correlated, respectively, with features $A_{1}$ and $A_{2}$ and the extremely shallow and broad features $\mathrm{A}_{3}$ and $\mathrm{A}_{4}$ in the $\mathrm{C}$ K-edge XANES spectrum of $\mathrm{Si} /(\mathrm{C}+\mathrm{Si})=$ 0.27 GNFs:Si, marked by vertical arrows in Figure 6a. The extreme shallowness and broadness of features $\mathrm{A}_{3}$ and $\mathrm{A}_{4}$ may be caused by disorderly distributed $\mathrm{Si}$ dopants and the contributions of the p-component projection of $\mathrm{Si} 3 \mathrm{~s}$ and $3 \mathrm{p}$ orbitals onto the X-ray absorbing $\mathrm{C}$ atoms, as shown by the PDOS of $\mathrm{Si} 3 \mathrm{~s}$ and $3 p$ states (Figure 6a). The PDOS of C 2p and Si 3sp states also suggest that the positive features within the ranges of 287-292 and 292-305 eV shown in Figure 2 are associated mainly with $\mathrm{C} 2 \mathrm{p}-\mathrm{Si} 3$ sp hybridized states. Calculated distinctive groups of features $T_{1}$ to $T_{4}$ seem to agree reasonably well with experimental features $A_{1}$ to $A_{4}$, which suggests that the positions of the Si dopants proposed in this study might actually be the case and that $\mathrm{Si}$ dopants form a Si-centered local tetrahedral bonding arrangement.

Figure $6 \mathrm{~b}$ shows the PDOS of $\mathrm{C} 2 \mathrm{p}$ states below $E_{\mathrm{f}}$ for GNFs: $\mathrm{Si}$ with $\mathrm{Si} /(\mathrm{C}+\mathrm{Si})=0.25$. Vertical arrows marked by $\mathrm{B}_{2}$ to $\mathrm{B}_{6}$ correspond to the five features in the $\mathrm{p}_{\sigma^{-}}$and $\mathrm{p}_{\pi^{-}}$-band regions shown in the spectrum of the $\mathrm{C} \mathrm{K} \alpha$ emission of the $\mathrm{Si} /(\mathrm{C}+\mathrm{Si})$ $=0.27$ sample shown in Figure 4. These features can be correlated with the five $\mathrm{E}_{2}$ to $\mathrm{E}_{6}$ features in the calculated PDOS of $\mathrm{C} 2 \mathrm{p}$ states shown in Figure $6 \mathrm{~b}$. In this figure, the most prominent feature, that is, feature $\mathrm{E}_{3}$, has been lined up with $\mathrm{B}_{3}$, which is the most prominent feature in the spectrum of the $\mathrm{C} \mathrm{K} \alpha$ emission of the $\mathrm{Si} /(\mathrm{C}+\mathrm{Si})=0.27$ sample. The calculated feature $E_{1}$ is not accounted for in the emission spectra. This discrepancy may be due to a calculation model with a highly ordered $\mathrm{Si}$ arrangement and the sensitive dependence of the spectra on the incident angle. Nevertheless, due to the broadness of the observed features, especially $\mathrm{B}_{5}$ and $\mathrm{B}_{6}$, and a $0.35 \mathrm{eV}$ resolution of XES data, one may say that the positions of calculated features $E_{2}$ to $E_{6}$ agree reasonably well with those in the $\mathrm{C} \mathrm{K} \alpha$ emission spectrum. The PDOS of Si $3 s$ and $3 p$ states below $E_{\mathrm{f}}$ are also shown in Figure 6b, which indicates that features $\mathrm{E}_{1}$ to $\mathrm{E}_{6}$ involve hybridization between $\mathrm{C} 2 \mathrm{p}$ and $\mathrm{Si}$ 3 sp states.

\section{Conclusion}

Present EFE, XANES, Raman, XPS, and XES measurements reveal that $\mathrm{Si}$ treatment alters drastically the electronic structure of GNFs. EFE measurements show that the turn-on electric field increases with the Si content, which suggests that Si dopants induce an increase of activation energy and a reduction of the metallic $\mathrm{sp}^{2}$-bonding arrangement. The shifts of the features in XANES, XPS, and Raman spectra show enhancement of diamond-like $\mathrm{sp}^{3}$ bonding in GNFs:Si. Comparison between experimental measurements and theoretical calculation suggests that $\mathrm{Si}$ dopants substitute $\mathrm{C}$ atoms and relax out of the graphene plane to form $\mathrm{sp}^{3}$ bonding with another $\mathrm{C}$ atom in the adjacent graphene plane in a Si-centered local tetrahedral atomic arrangement.

Acknowledgment. W.F.P. would like to thank the National Science Council of Taiwan for financially supporting this research under Contract No. NSC 96-2112-M032-012-MY3. The Advanced Light Source is supported by the U.S. Department of Energy under Contract No. DE-AC02-05CH11231.

\section{References and Notes}

(1) Wu, Y. H.; Qiao, P. W.; Chong, T. C.; Shen, Z. X. Adv. Mater. 2002, 14,64

(2) Ando, Y.; Zhao, X.; Ohkohchi, M. Carbon 1997, 35, 153.

(3) Shang, N. G.; Au, F. C. K.; Meng, X. M.; Lee, C. S.; Bello, I.; Lee, S. T. Chem. Phys. Lett. 2002, 358, 187.

(4) Wang, J. J.; Zhu, M. Y.; Outlaw, R. A.; Zhao, X.; Manos, D. M.; Holloway, B. C. Carbon 2004, 42, 2867.

(5) Hiraki, H.; Jiang, N.; Wang, H. X.; Hiraki, A. J. Phys. IV 2006, 132,111 .

(6) Nishimura, K.; Jiang, N.; Hiraki, A. IEICE Trans. Electron. E 2003, 86,821

(7) (a) Shang, N. G.; Papakonstantinou, P.; McMullan, M.; Chu, M.; Stamboulis, A.; Potenza, A.; Dhesi, S. S.; Marchetto, H. Adv. Funct. Mater. 2008, 18, 3506. (b) Abbas, G. A.; Papakonstantinou, P.; McLaughlin, J. A.; Weijers-Dall, T. D. M.; Elliman, R. G.; Filik, J. J. Appl. Phys. 2005, 98, 103505.

(8) Guo, G.; Wang, F.; Sun, H.; Zhang, D. Int. J. Quantum Chem. 2008, 108, 203

(9) Baierle, R. J.; Fagan, S. B.; Mota, R.; da Silva, A. J. R.; Fazzio, A. Phys. Rev. B 2001, 64, 085413.

(10) Fagan, S. B.; Mota, R.; Baierle, R. J.; da Silva, A. J. R.; Fazzio, A. Diamond Relat. Mater. 2003, 12, 861.

(11) Song, C.; Xia, Y.; Zhao, M.; Liu, X.; Li, F.; Huang, B.; Zhang, H.; Zhang, B. Phys. Lett. A 2006, 358, 166.

(12) Ray, S. C.; Palnitkar, U.; Pao, C. W.; Tsai, H. M.; Pong, W. F.; Lin, I.-N.; Papakonstantinou, P.; Chen, L. C.; Chen, K. H. J. Appl. Phys. 2008, 104, 063710

(13) Ray, S. C.; Pao, C. W.; Tsai, H. M.; Chiou, J. W.; Pong, W. F.; Chen, C. W.; Tsai, M.-H.; Papakonstantinou, P.; Chen, L. C.; Chen, K. H. Appl. Phys. Lett. 2007, 91, 202102.

(14) Ray, S. C.; Pao, C. W.; Tsai, H. M.; Chiou, J. W.; Jan, J. C.; Krishna Kumar, K. P.; Pong, W. F.; Tsai, M.-H.; Wang, W.-J.; Hsu, C.-J.; Okpalugo, T. I. T.; Papakonstantinou, P.; McLaughin, J. A. Appl. Phys. Lett. 2004, 85,4022 .

(15) Terekhov, V. A.; Terukov, E. I.; Trapeznikova, I. N.; Kashkarov, V. M.; Kurilo, O. V.; Turishchev, S. Yu.; Golodenko, A. B.; Domashevskaya, É. P. Semiconductors 2005, 39, 830

(16) Chang, Y. K.; Hsieh, H. H.; Pong, W. F.; Tsai, M.-H.; Lee, K. H.; Dann, T. E.; Chien, F. Z.; Tseng, P. K.; Tsang, K. L.; Su, W. K.; Chen, L. C.; Wei, S. L.; Chen, K. H.; Bhusari, D. M.; Chen, Y. F. Phys. Rev. B 1998, $58,9018$. 
(17) Pacilié, D.; Papagno, M.; Rodríguez, A. F.; Grioni, M.; Papagno, L.; Girit, C. Ó.; Meyer, J. C.; Begtrup, G. E.; Zettl, A. Phys. Rev. Lett. 2008, 101, 066806 .

(18) Huang, J.-R.; Lin, J.-Y.; Chen, B.-H.; Tsai, M.-H. Phys. Status Solidi B 2008, 245, 136

(19) Ferrari, A. C.; Robertson, J. Phys. Rev. B 2001, 64, 075414.

(20) Choi, S.; Park, K. H.; Lee, S.; Koh, K. H. J. Appl. Phys. 2002, 92, 4007.

(21) Pedio, M.; Borgatti, F.; Giglia, A.; Mahne, N.; Nannarone, S.; Giovannini, S.; Cepek, C.; Magnano, E.; Bertoni, G.; Spiller, E.; Sancrotti, M.; Giovanelli, L.; Floreano, L.; Gotter, R.; Morgante, A. Phys. Scr., T 2005, 115, 695 .

(22) Skytt, P.; Glans, P.; Mancini, D. C.; Guo, J.-H.; Wassdahl, N.; Nordgren, J.; Ma, Y. Phys. Rev. B 1994, 50, 10457.
(23) Carlisle, J. A.; Shirley, E. L.; Hudson, E. A.; Terminello, L. J.; Callcott, T. A.; Jia, J. J.; Ederer, D. L.; Perera, R. C. C.; Himpsel, F. J. Phys. Rev. Lett. 1995, 74, 1234.

(24) Dai, J.; Yuan, J.; Giannozzi, P. Appl. Phys. Lett. 2009, 95, 232105.

(25) Table of Periodic Properties of the Elements; Sargent-Welch Scientific Company: Skokie, IL, 1980.

(26) Lide, D. R., Ed. CRC Handbook of Chemistry and Physics, 80th ed.; CRC Press: Boca Raton, FL, 1999-2000; pp 4-150.

(27) Kasowski, R. V.; Tsai, M.-H.; Rhodin, T. N.; Chambliss, D. D. Phys. Rev. B 1986, 34, 2656.

(28) Chadi, D. J.; Cohen, M. L. Phys. Rev. B 1973, 8, 5747.

(29) Monkhorst, H. J.; Pack, J. D. Phys. Rev. B 1976, 13, 5188.

JP9121563 